\title{
Vehicle appearance feature recognition based on image
}

\author{
Deyang Shao ${ }^{1, \mathrm{a}}$, Chao $\mathrm{Xu}^{2, \mathrm{~b}}$, Shixian $\mathrm{Luo}^{2, \mathrm{c}}$, Bo Feng ${ }^{2, \mathrm{~d}}$, and Long Jiao ${ }^{2, \mathrm{e}}$
}

${ }^{1}$ Anhui Engineering Laboratory of Agro-Ecological Big Data, Anhui University, Hefei, 230601, China;

${ }^{2}$ Key Laboratory of Intelligent Computer \& Signal Processing, Ministry of Education, Anhui

University, Hefei 230601, China.

a782740870@qq.com, braymagpie@163.com,

c136946382@qq.com, ${ }^{\mathrm{d}} 1160596116 @ q q . c o m .{ }^{\mathrm{e}} 386950757 @ q q . c o m$

Keywords: Feature information, ORB, RANSAC, Affine transformation.

\begin{abstract}
Now, the highway toll system still uses a single license plate recognition, this method has a problem of inaccurate identification. For this kind of situation, this paper put forward to increase the appearance of the vehicle feature information and can improve the accuracy of recognition. In this paper, we adopt the ORB algorithm to extract the exterior feature information of the vehicle and two-way matching、RANSAC algorithms to remove mismatching points. At the same time, we continue to iteration the scale parameter of the affine transformation and rotation angle at the matching point as a kind of judgment, which improves the robustness of the algorithm.
\end{abstract}

\section{The introduction}

With the development of social economy and people living standard rise, more and more cars are running on the highway. How fast and accurate identification of vehicles, to the high-speed toll Department has brought the problem. At present the vehicle identification is still using the license plate recognition method on highway, it cause some truck drivers adopt the method of deck to escape tolls. This has brought great losses to the highway management department, in order to reduce the loss, this paper presents a new algorithm based on vehicle appearance recognition.

The classical algorithm has SIFT algorithm, although it is relatively mature and stable algorithm, however, due to the large amount of computation of SIFT algorithm and can not reach the requirements of real-time. If we adopt the SURF algorithm to extract the local features of the vehicle, due to the bayonet camera resolution and light conditions is different of the highway toll station. We consider the above situations, so we adopt the ORB algorithm.

For the error matching, many scholars of domestic and foreign have put forward many methods to eliminate the false matching points. Li Zhi proposed a feature point matching based on matching intensity. This algorithm can eliminate the false matching points by using the matching support value, which can eliminate the one-to-many false matching conditions. Yan Weidong put forward a kind of based on partial least squares (PLS) of the scale invariant feature transform (SIFT) error matching correction methods. This method uses the position information of the feature points after SIFT matching and re-described by PLS. We use the definition of impact function will affect the large feature points to remove, it can effectively remove the false match. Lei Yuzhen put forward random sample consensus (RANSAC) algorithm. He is the use of the mark point of the space feature invariance to realize the automatic matching of the mark points and to eliminate the false matching points.

\section{Algorithm theory}

As shown in Figure 1. The first, we get the image preprocessing, it can improve the image contrast and clarity. Then, we use ORB algorithm to extract the vehicle's appearance feature information after preprocessed image. The feature information have car models, logo, car gate features etc. Next, the coarse matching was performed based on entrance and export vehicles' feature information. Then, we use the improved RANSAC algorithm to remove false matching 
points and statistics on the matching points and then compared with the threshold value as a judgment strategy. The last, affine transformation is used to identify the vehicle matching again as the secondary judgment strategy.

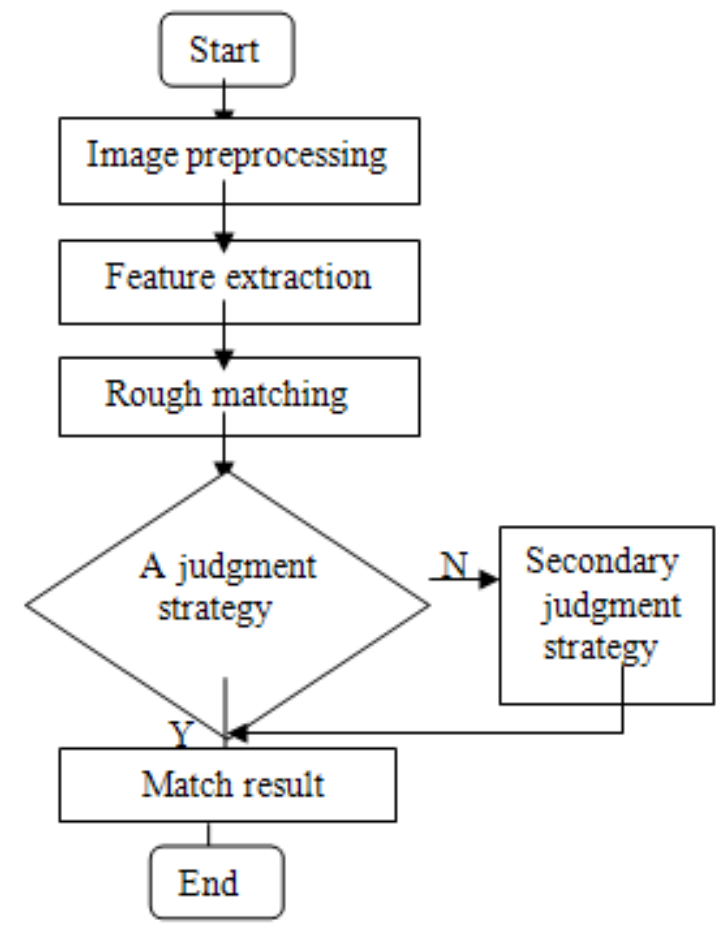

Figure 1

\subsection{Video preprocessing.}

Due to bayonet of each highway toll station situation is more complex, special light conditions. All these lead to obtain image resolution is not high and serious influence the subsequent vehicle identification effect. So we need to do image preprocessing and improve the clarity of the image. Through the study of various bayonet video, we found that we obtain the image is "dusky" feeling, it is similar to the situation of the fog. So we adopt the principle of image to fog to enhance the image, this reduces some interference for the next vehicle identification, as shown in Figure 2.

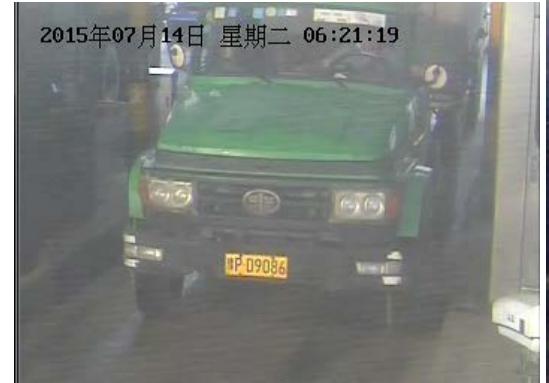

Original

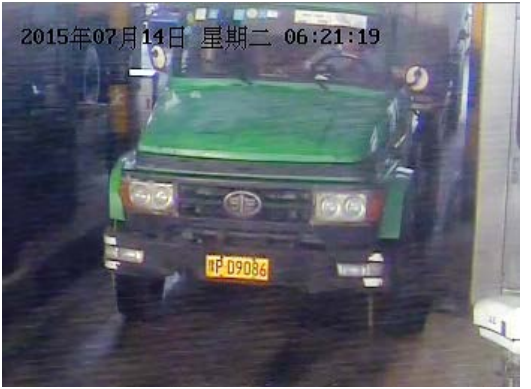

After pretreatment

Figure 2

\subsection{Vehicle feature extraction.}

In every day, the number of vehicles through the toll station of highway is relatively large, this causes that the real-time requirements of the algorithm is relatively high. So in this paper, we adopt the ORB algorithm to extract the feature points. The ORB algorithm firstly uses the FAST algorithm to detect the feature points, and then use the BRIEF algorithm to describe the feature points. Because the original FAST algorithm have no direction. In ORB, we use the gray mass center method to give the feature points to the main direction, this is called Oriented-FAST. We calculated with feature points as the center and radius $r$ of the circular neighborhood within the scope of the gray centroid moment. We define the vector as the main direction from the center of the feature point to the center of the center of mass.

Definition of gray moment: $x, y \in[-r, r]$ 


$$
\mathrm{m}_{\mathrm{pq}}=\sum \mathrm{x}^{\mathrm{p}} \mathrm{y}^{\mathrm{q}} \mathrm{I}(\mathrm{x}, \mathrm{y})
$$

$\mathrm{I}(\mathrm{x}, \mathrm{y})$ as grey value of the center point $(\mathrm{x}, \mathrm{y})$

position of the center of mass mass:

$$
\mathrm{C}=\left(\frac{\mathrm{m}_{10}}{\mathrm{~m}_{00}}, \frac{\mathrm{m}_{01}}{\mathrm{~m}_{00}}\right)
$$

The main direction:

$$
\theta=\alpha \tan 2\left(\mathrm{~m}_{01}, \mathrm{~m}_{10}\right)
$$

The ORB using Rotated-BRIEF algorithm, which makes the feature descriptor with rotation invariance. The BRIEF algorithm is simple and fast. Its basic idea is that the field of image can be expressed by a relatively small amount of intensity. The BRIEF descriptor is a bit string description of an image patch constructed from a set of binary intensity tests.

$$
\tau(\mathrm{p} ; \mathrm{x}, \mathrm{y}):=\left\{\begin{array}{cc}
1 & \mathrm{p}(\mathrm{x})<\mathrm{p}(\mathrm{y}) \\
0 & \text { other }
\end{array}\right.
$$

where $\mathrm{p}(\mathrm{x})$ is the intensity of $\mathrm{p}$ at a point $\mathrm{x}$.

We choose for $\mathrm{N}$ position, only the definition of binary criterion, the BRIEF descriptors for the n-dimensional binary bit string:

$$
\mathrm{f}_{\mathrm{N}}(\mathrm{p}):=\sum_{1 \leq \mathrm{i} \leq \mathrm{N}} 2^{\mathrm{i}-1} \tau\left(\mathrm{p} ; \mathrm{x}_{\mathrm{i}}, \mathrm{y}_{\mathrm{i}}\right)
$$

$\mathrm{N}=128,256$ or 512, in this paper, we take 256. The BRIEF itself does not have rotation invariance. The solution strategy adopted by ORB is to define a $2 \mathrm{n}$ matrix in the position ( $\mathrm{x}, \mathrm{y})$ for any $\mathrm{n}$ binary $\tau$ criterion. At same time, the principal direction $\theta$ and rotation matrix $\mathrm{R}_{\theta}$ are obtained by using the gray centroid method and the resulting is $S_{\theta}$.

$$
\begin{aligned}
& S=\left(\begin{array}{lll}
x_{i} & \cdots & x_{n} \\
y_{i} & \cdots & y_{n}
\end{array}\right) \\
& S_{\theta}=R_{\theta} S
\end{aligned}
$$

Get the final Rotated-BRIEF descriptor is expressed as:

$$
g_{n}(p, \theta):=f_{N}(p) \mid\left(x_{i}, y_{i}\right) \in S_{\theta}
$$

\subsection{Vehicle matching.}

We use the Hamming distance as the feature point matching strategy. Firstly, we use the feature information of the vehicle(the entrance to the vehicle) which has been extracted to match the feature information of the vehicle at this time. Since the two vehicles' features are not captured in the same scene, there is a light, environmental and other factors will lead to a lot of wrong matching pairs. Now, we have many ways to eliminate the error matching, for example, the distance ratio method. But it does not play a very good effect for those who appear to be relatively concentrated in the miss match. So we use two-way matching method, the idea is: according to the feature point matching is one-to-one relationship, first we use the feature point set of target image and feature point set of the matching image to do a nearest neighbor matching. Then, we use the feature point set of the matching image and feature point set of target image to do a nearest neighbor matching again. We choose the intersection of the two matching data, so that excluding non intersection points, those non intersection points is the point of error matching, but still exist some wrong matching points in these intersection points.

\subsection{Judgment strategy.}

Aiming at the error matching points in the intersection of the above, we use the RANSAC algorithm to remove the false matching points. The RANSAC algorithm is a kind of classic algorithm to eliminate the error matching, which has the advantages of high matching precision, strong robustness and so on. By estimating the optimal model parameters, we can satisfy the maximum number of points in the model parameters. But the RANSAC algorithm has some defects, it only has a certain probability to obtain the optimal transformation parameters. In order to reduce the probability of false judgment, we choose to intercept a series of images in the video, such as 20, and then repeated using the above algorithm iterative computation as a judgment strategy. If the proportion of the number of matching pairs is greater than $18 \%$, then the vehicle is judged to be the correct vehicle, otherwise the two judgment strategy is adopted.

In this paper, we use RANSAC algorithm to estimate an optimal should a single transformation matrix $\mathrm{H}_{3 \times 3}$. 


$$
S\left[\begin{array}{c}
x^{\prime} \\
y^{\prime} \\
1
\end{array}\right]=\left[\begin{array}{lll}
h_{11} & h_{12} & h_{13} \\
h_{21} & h_{22} & h_{23} \\
h_{31} & h_{32} & h_{33}
\end{array}\right]\left[\begin{array}{l}
x_{1} \\
y \\
1
\end{array}\right]
$$

Among them, $S$ is a scale factor, we make $h_{33}=1$ to carry out the normalization of this unit matrix, so the matrix $\mathrm{H}$ has 8 unknown parameters, it needs at least 4 matching points to solve the equation.

The RANSAC algorithm uses the following formula to estimate the number of iterations.

$$
\mathrm{k}=\frac{\log (1-\mathrm{p})}{\log \left(1-\mathrm{w}^{\mathrm{m}}\right)}
$$

Where $\mathrm{m}$ represents the minimum number of samples required to estimate the model, in this paper, $\mathrm{m}=4, \mathrm{p}$ is algorithm can give the probability of the optimal model, $\mathrm{w}$ represents the ratio of matching points, and w is constantly updated in the iterative operation. Figure 2 shows the results of tests conducted on 20 trucks. We extracted 200 feature points for matching, the results are shown in fig 2.

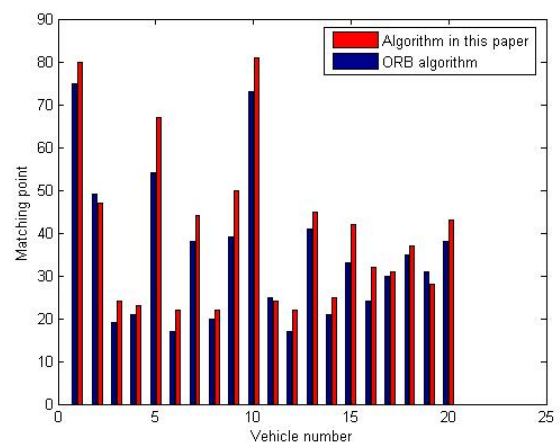

Figure 2 comparison of matching results

For the camera position caused by poor access to images in the vehicle the appearance is not complete. We adopt the strategy of a judgment will lead to the result of an error, so we adopt the strategy of secondary judgment.

We use the least squares method to estimate an optimal affine transformation matrix on the set of matching pairs. We calculate the affine transformation matrix of the scale ratio and the average value whether in the $[0.7,1.3]$ and affine angle in the $[-30,30]$. If both fall into the specified area, we judged to be the right vehicle, otherwise we are judged as illegal vehicles.

$$
[A \mid b]=\left[\begin{array}{lll}
a_{11} & a_{12} & b_{1} \\
a_{21} & a_{22} & b_{2}
\end{array}\right]=\left[\begin{array}{ccc}
s_{x} \cos \theta & -s_{x} \sin \theta & t_{x} \\
s_{y} \sin \theta & s_{y} \cos \theta & t_{y}
\end{array}\right]
$$

The size of the affine transformation matrix is $2 \times 3$, which is composed of the rotation scale transformation matrix $A_{2 \times 2}$ and the translation transformation matrix $b_{2 \times 1}$. Where $s_{x}, s_{y}$ represents the size of the scale transformation, $\theta$ represents the rotation angle and $t_{x}, t_{y}$ represents the translation amount.

The calculation method of scale transform and the rotation angle:

$$
s_{\mathrm{x}}=\sqrt{\mathrm{a}_{11}^{2}+\mathrm{a}_{12}^{2}} \quad \mathrm{~s}_{\mathrm{y}}=\sqrt{\mathrm{a}_{21}^{2}+\mathrm{a}_{22}^{2}} \quad \theta=\mathrm{a} \tan 2\left(-\mathrm{a}_{12}, \mathrm{a}_{11}\right)
$$

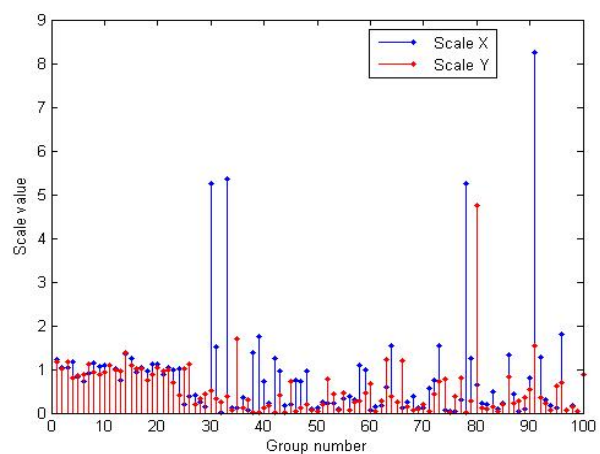

Figure 3 scale of affine transformation

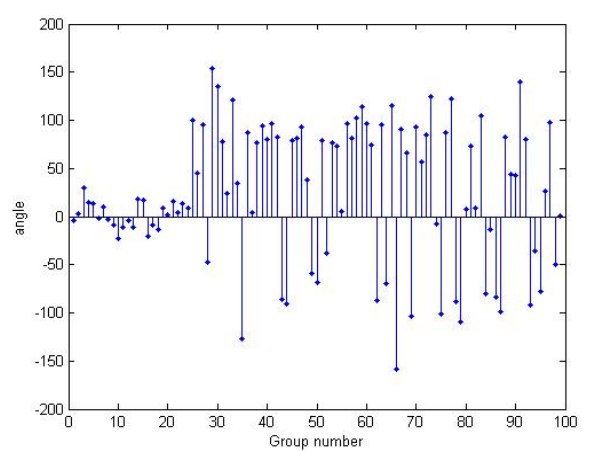

Figure 4 angle of affine transformation

We carry on the statistics of the 100 experimental data, including the first 25 of the same car data, 
after the 75 are not the same car data. We find that the scale of the affine transformation of the correct vehicle is mostly concentrated in the $[0.7,1.3]$, and the rotation angle is concentrated in the $[-30,30]$.

\section{The experimental results}

In this paper, the algorithm platform constructed by VS2008 and OpenCV2.4.3 version. The computer platform configuration for 32-bit operating systems, the memory is 4Gb and the CPU is 3.1 GHz. We use the standard $\mathrm{C} / \mathrm{C}++$ language programming to realize. We took 100 data to carry out experiments, including the first 25 of the same car data, after the 75 are not the same car data. During the match, we choose 20 consecutive images of the export video to match and take the average value as the matching results. The matching results are shown in the following table one.

Table 1 Accuracy

\begin{tabular}{|c|c|c|c|}
\hline & The same car & Not the same car & Correct rate \\
\hline Total & 25 & 75 & \\
\hline Affine transformation & 1 & 3 & $96 \%$ \\
\hline No affine transformation & 5 & 2 & $93 \%$ \\
\hline
\end{tabular}

\section{The conclusion}

This paper is aimed at the problem of the highway toll station's charging mechanism depends on the license plate recognition. We propose to increase the appearance feature information of vehicle identification. Through the study of the experimental verification of a large number of toll station monitoring video. We conclude that the method of this paper improves the accuracy and robustness of the recognition to a certain extent. But there are some problems, for example, in dense fog, night, and other conditions under the harsh environment. The results we obtained are not very good, it will be our next research direction.

\section{Acknowledgment}

The project is supported by the science and technology project of Anhui Province: Research and development and application of key technology of integrated intelligent system for agricultural sensing and control system (1604a0702016).

\section{References}

[1]. ZENG Jun, LI Dehua. Color image SUSAN edge detection method. Computer Engineering and Applications. 2011. 47(15), 194-196.

[2]. SUN Rui, WANG Jing-Jing. Vehicle identification method based on kernel K-SVD and sparse representation. Pattern recognition and artificial intelligence. 2014.27(5), 435-442.

[3]. Xu Jiajia, Zhang Ye, Zhang He. Fast image registration algorithm based on improved Harris-SIFT operator. Journal of Electronic Measurement and Instrumentation. 2015. 29(1), 48-54.

[4]. HOU Y, ZHOU S L, LEI L, et al. Fast fully affine invariant image matching based on ORB. Compute Engineering \& Science 2014. 36(2), 303-310.

[5]. RUAN Xiaoli, CHEN Qinghu, QIU Yiming, YAN Yuchen. SIFT false matching point elimination and image retrieval based on invariant factor. Infrared Technology. 2015. 37(7), 561-565.

[6]. CHEN Jianpeng, BI Duyan, ZHANG Shengchong, Fast defogging method based on dark channel theory. Computer Engineering and Design. 2014. 35(6), 2047-2051. 